\title{
Aggregative Spinnennetze - weitere Funde in Deutschland und mögliche Erklärungen
}

Peter JÄGER

\begin{abstract}
Aggregative spider webs - further records in Germany and possible explanations. In contrast to social spider webs aggregative spider webs are defined as incidental and non-functional. Three new records of large (3-10 meter) aggregative spider webs in Germany are presented. In one case (cut fir tree) 11 spider species were recorded from the same web. Two main reasons for the occurrence of large aggregative webs are hypothesized: 1. Optimal (weather) conditions for ballooning behaviour may cause a mass occurrence of spiders, which are able to cover vegetation structures with their draglines. 2. Disturbances in the spider habitat (e.g. cutting trees) may cause a movement of spiders to higher (then absent) strata, which may cover also vegetation with huge webs. In both cases spiders may aggregate on the surface of vegetation structures and webs are more dense at their higher points. A parasocial aggregation cannot be excluded, but according to the new observations this seems to be unlikely.
\end{abstract}

Key words: aggregative spider webs, canopy dwelling species, ballooning behaviour

\section{AGGREGATIVE SPINNENNETZE - BEGRIFFSKLÄRUNG}

Spinnen sind im allgemeinen Einzelgänger. Fast alle netzbauenden Spinnen bauen daher ein eigenes Fang- oder Wohnnetz. Wie so oft, gibt es von dieser Regel Ausnahmen. Die wohl bekannteste sind die sozialen Spinnen, die z.T. sehr grosse Gemeinschaftsnetze weben und in diesen mit verschiedenen Generationen zusammen leben, jagen, fressen und sich fortpflanzen. Beispiele sind aus verschiedenen Familien bekannt: Eresidae (Stegodyphus spp.), Agelenidae (Agelena consociata DENIS, 1965), Theridiidae (Anelosimus eximius (KEYSERLING, 1884)) oder Araneidae (Cyrtophora sp., Eriophora sp.). Die Netze der vorstehenden Arten bezeichne ich als Gemeinschaftsnetze oder soziale Netze, weil sie gemeinschaftlich angelegt und benutzt werden. Sie haben eine Funktion im Lebenszyklus der Spinnen und werden regelmäßig von Individuen dieser Arten gewebt. 
Der Begriff ,aggregative Spinnennetze“ dagegen bezeichnet Netze, die zwar de facto von mehreren Spinnen gefertigt wurden, aber weder als Fangnetz dienen noch für irgendeine andere soziale oder parasoziale Tätigkeit. Wie weiter unten beschrieben, können diese Netze auch von verschiedenen Arten hergestellt werden. Aggregative Netze werden im Gegensatz zu Gemeinschaftsnetzen zufällig und nicht zu einem bestimmten Zweck hergestellt. Grundsubstanz stellt der Sicherheitsfaden(„dragline“) der einzelnen Spinnen dar. Aggregative Netze werden nach den bisherigen Kenntnissen nicht regelmäßig hergestellt.

Ein Beispiel eines solchen aggregativen Netzes wurde von HEER (1997) aus der Schweiz beschrieben (Kanton Aargau: Oberentfelden). Ein Haufen von Fichtenzweigen auf einer Waldlichtung war mit Spinnweben überdeckt und Tausende von Individuen von Troxochrus nasutus SCHENKEL, 1925 liefen auf dem Netz umher. HEER bezeichnet dieses Phänomen als ,parasoziales Verhalten“.

Die im weiteren geschilderten, neuen Funde geben Erkenntnisse über das Entstehen solcher Netze und geben Anlass zu Zweifel, dass es sich um (parasoziale) Gemeinschaftsnetze handelt.

\section{NEUE FUNDE AGGREGATIVER NETZE IN DEUTSCHLAND}

1. Markgröningen, April 2001: Auf einem Trockenrasen wurden Spinnennetze beobachtet, die über vertrocknete vorjährige Blütenstände von Umbelliferen gespannt waren. Die längste Ausdehnung des Gesamtnetzes betrug sechs Meter (MORGENSTERN, pers. Mitt.). Ein Individuum wurde fotografiert, einige andere (offensichtlich konspezifische) Tiere beobachtet. Bei dem fotografierten Tier handelt es sich um ein subadultes Männchen einer Linyphiide, Araneide oder Theridiide, welches anscheinend versucht, von einem erhöhten Punkt aus, einen Seidenfaden in den Wind zu entlassen (Abb. 1). Das sonnige Wetter und der blaue Himmel beides auf den Fotos dokumentiert - sprechen für eine Ballooning-Aktivität. Belegexemplare oder detailliertere Beobachtungen liegen nicht vor. 


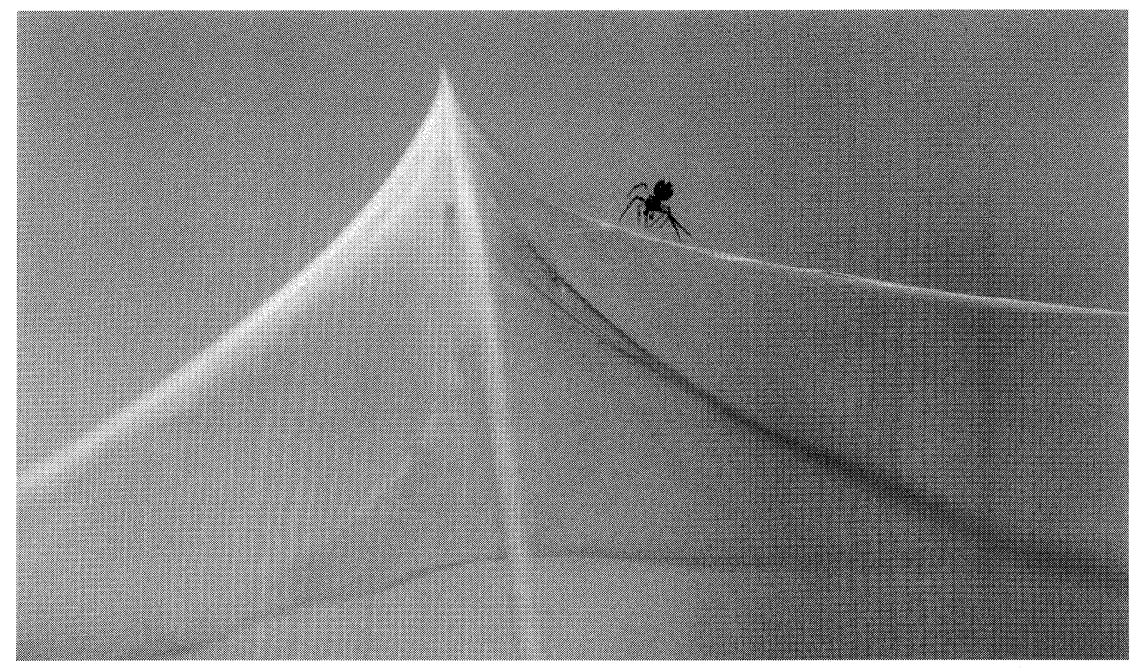

Abbildung 1: Aggregatives Spinnennetz, Trockenrasen nahe Markgröningen, April 2001. Subadultes (?) Männchen bei der Startphase für aeronautisches Verbreitungsverhalten (Foto: W. MORGENSTERN).

Figure 1: Aggregative spider web, dry grassland near Markgröningen, April 2001. Subadult (?) male in the starting stage of the ballooning behaviour (photo: W. MORGENSTERN).

2. Hochheim, Weinbergsbrache zwischen Hochheim und Mainz-Kostheim. Ende Oktober 2001. Über zwei Haufen von geschredderten Gartenabfällen (jeweils ca. 10 Meter lang und an den höchsten Stellen 1.80 Meter hoch) wurde ein Gespinst aus Seidenfäden beobachtet (Abb. 2). Pilze und keimende Pflanzen hoben das robuste Gespinst an einigen Stellen empor. Den z.T. gärenden Abfällen (Abfälle vom Weinbau?) entwichen Insekten (z.B. Drosophila sp.). Auf dem Spinnennetz befanden sich Tausende von kleinen Spinnen (KUHN, pers. Mitt, Abb. 3-4). Die Determination von zwei Männchen aus einer Probe des Netzes ergab die Art Ostearius melanopygius (O. P.-CAMBRIDGE, 1879). Die Probe war erst nach der teilweisen Zerstörung des bereits verwitterten Netzes genommen worden. $\mathrm{Zu}$ diesem Zeitpunkt befanden sich ausschließlich tote Exemplare im Gewebe. Ob noch andere Arten beteiligt gewesen waren, konnte nicht mehr 


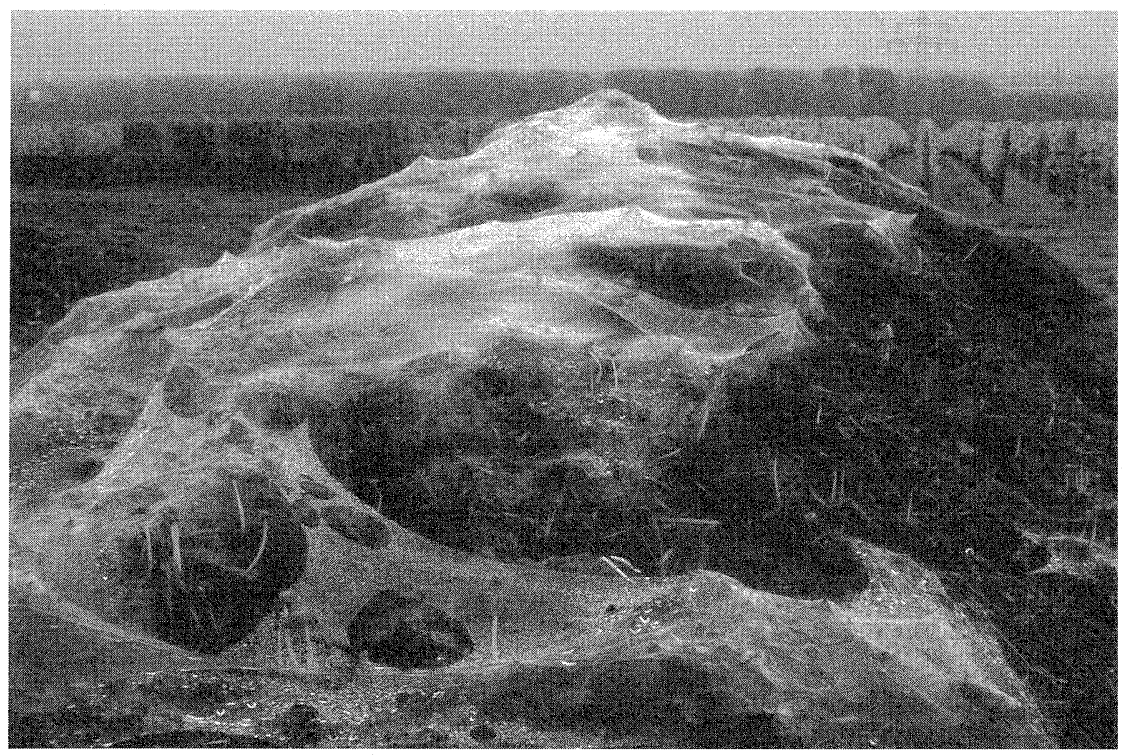

Abbildung 2: Aggregatives Spinnennetz von Ostearius melanopygius, Weinbergsbrache zwischen Hochheim und Mainz-Kostheim, Ende Oktober 2001. (Foto: R. KUHN).

Figure 2: Aggregative spider web of Ostearius melanopygius, vineyard fallow between Hochheim and Mainz-Kostheim, end of October 2001. (photo: R. KUHN).

festgestellt werden. Die auf den Fotos dokumentierten Spinnen zeigen jedoch bis auf wenige Ausnahmen die für Ostearius typische rötliche Färbung des Opisthosomas. Einzelne gänzlich schwarz gefärbte Exemplare könnten ebenfalls zu dieser Art gehören, da auch solche Ausnahmen aus der Literatur bekannt sind (ROBERTS 1987, HEIMER \& NENTWIG 1991). Einzelne Spinnen auf der Netzdecke zeigten die ZehenspitzenPhase (,tiptoe-behavior") des Ballooning-Verhaltens (Abb. 4). 
3. Mainz, Draisberghof, 3.12.2001: Auf einem Haufen von Zweigen, die am 1.12.2001 von einer frisch gefällten Tanne (Abies sp., ca. 8 m Höhe) abgeschnitten worden waren und direkt danach auf einer Ackerfläche von etwa drei mal vier Meter gelagert wurden, wurde ein Netz beobachtet, welches sich beinahe über die ganze Fläche des Haufens erstreckte. Auf bzw. unter dem Netz bewegten sich Hunderte von Spinnen (Abb. 5-6). Dabei war das Gewebe an erhöhten Punkten deutlich stärker ausgebildet bzw. mit mehr Individuen besetzt. Bei einer Probennahme wurden nahezu alle Netzbereiche samt Spinnen abgesammelt. Dabei wurden nicht alle Individuen erfasst, einige ließen sich zu Boden fallen bzw. wurden vom Wind weggeweht.

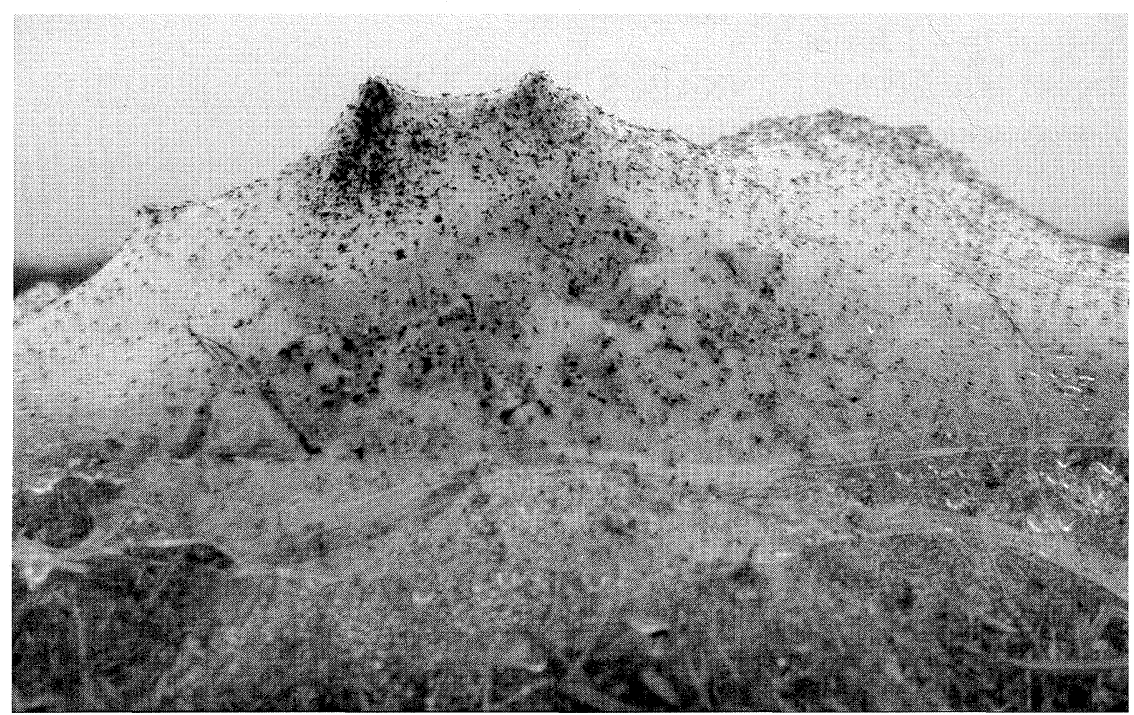

Abbildung 3: Spinnen (Ostearius melanopygius) auf aggregativem Netz, Weinbergsbrache zwischen Hochheim und Mainz-Kostheim, Ende Oktober 2001. (Foto: R. KUHN).

Figure 3: Spiders (Ostearius melanopygius) on aggregative web, vineyard fallow between Hochheim and Mainz-Kostheim, end of October 2001. (photo: R. KUHN). 
Die Bestimmung der Spinnen brachte folgendes Ergebnis (Arten geordnet nach Häufigkeit mit Angabe der Individuenanzahl; Summe $=590$ Individuen):

Anelosimus vittatus (C. L. KOCH, 1836) - 487 (davon 5 adulte Weibchen, zahlreiche subadulte Weibchen und Männchen, sowie verschiedene Altersstadien von juvenilen Spinnen)

Philodromus sp. - 67 (darunter subadulte Männchen und verschiedene Altersstadien juveniler Spinnen)

Theridion sp. (tinctum?) - 16 Juvenile

Lathys sp. (humilis?) - 10 (davon 1 subadultes Männchen)

Araniella sp. - 2 Juvenile

Clubiona sp. - 2 (davon 1 subadultes Männchen)

Theridiidae sp. - 2 Juvenile

Misumena vatia (CLERCK, 1757) - 1 Juvenile

Salticus sp. - 1 Juvenile

Segestria senoculata (LINNAEUS, 1758) - 1 Juvenile

Tetragnatha sp. - 1 Juvenile

Das Wetter war über die gesamte Beobachtungszeit bedeckt bis regnerisch mit leichtem bis mäßig starkem Wind. Alle Spinnen saßen oder bewegten sich auf oder unter der Netzdecke. Kleinere Stadien von Anelosimus vittatus versuchten, im leichten Wind mit dem Fadenfloß zu starten, was aber nicht gelang. Nachdem das Netz beim Sammeln zerstört worden war, konnten an den Folgetagen (4./5.12.2001) neue (kleinere) Netze mit etlichen Spinnen (Anelosimus) beobachtet werden. Am 6.12.2001 zerstörten stürmische Winde den Großteil der Netze. Spinnen waren nur noch vereinzelt an geschützten Stellen zu sehen. Im Februar 2002 waren immer noch einige Individuen von Anelosimus vittatus $\mathrm{zu}$ beobachten.

Die Tanne hatte über 25 Jahre alleinstehend in einem Vorgarten gestanden. Die einzigen in der Nähe stehenden Gehölze waren eine Magnolie (Magnolia sp.) und eine Fliederhecke (Syringa sp.) (beides ca. 10-15 Meter entfernt). In einem Garten hinter dem Haus standen zahlreiche Nadelbäume (Larix sp., Abies sp., Picea sp., Pinus sp., Taxus sp.) (ca. 20-40 Meter entfernt). 


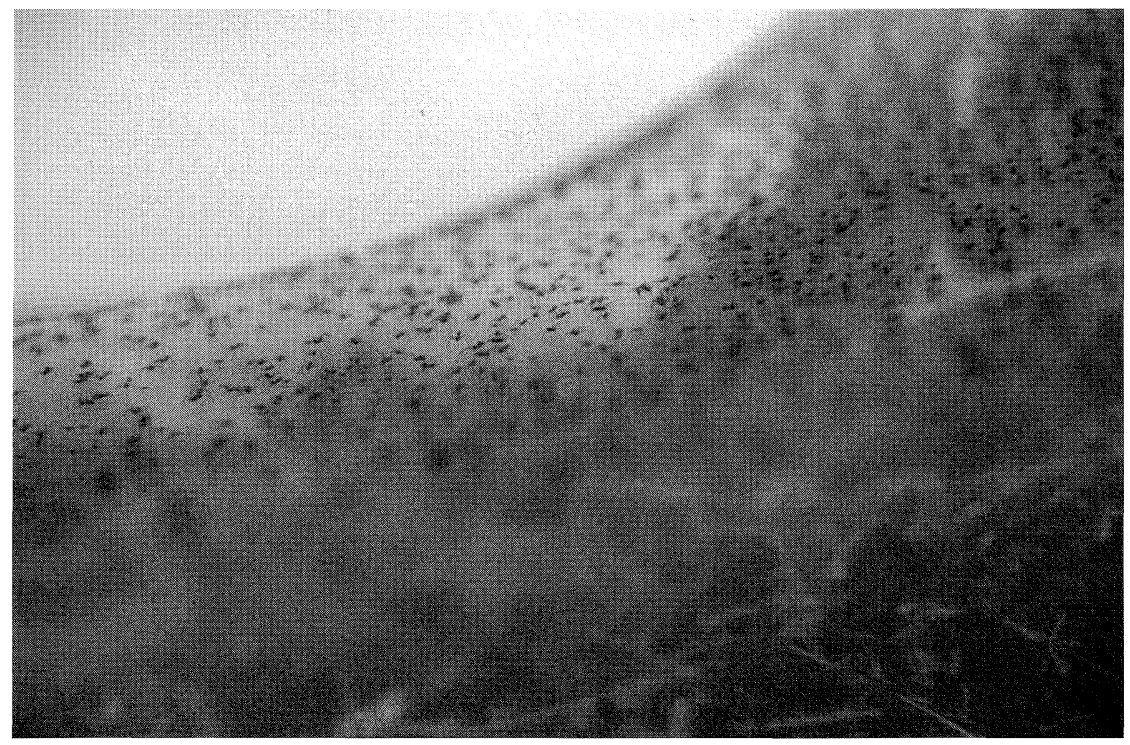

Abbildung 4: Spinnen (Ostearius melanopygius) auf aggregativem Netz, Weinbergsbrache zwischen Hochheim und Mainz-Kostheim, Ende Oktober 2001. Einzelne Spinnen zeigen Zehenspitzen-Phase des BallooningVerhaltens (Foto: R. KUHN).

Figure 4: Spiders (Ostearius melanopygius) on aggregative web, vineyard fallow between Hochheim and Mainz-Kostheim, end of October 2001. Single spiders showing tiptoe-behaviour before ballooning (photo: R. KUHN).

\section{DISKUSSION}

Die oben beschriebenen neuen Fälle von aggregativen Spinnennetzen besitzen eine Gemeinsamkeit: alle wurden von mehreren, wenn nicht vielen Hunderten oder Tausenden Individuen gesponnen. In allen Fällen scheint der Sicherheitsfaden der einzelnen Spinnen den Baustoff für die Gewebe darzustellen.

Das Netz aus Markgröningen deutet auf einen typischen Fall von Ballooning-Aktivität hin. Bei schönem Wetter mit leichten Winden versuchen Spinnen, sich neue Lebensräume zu erschließen, indem sie sich an 


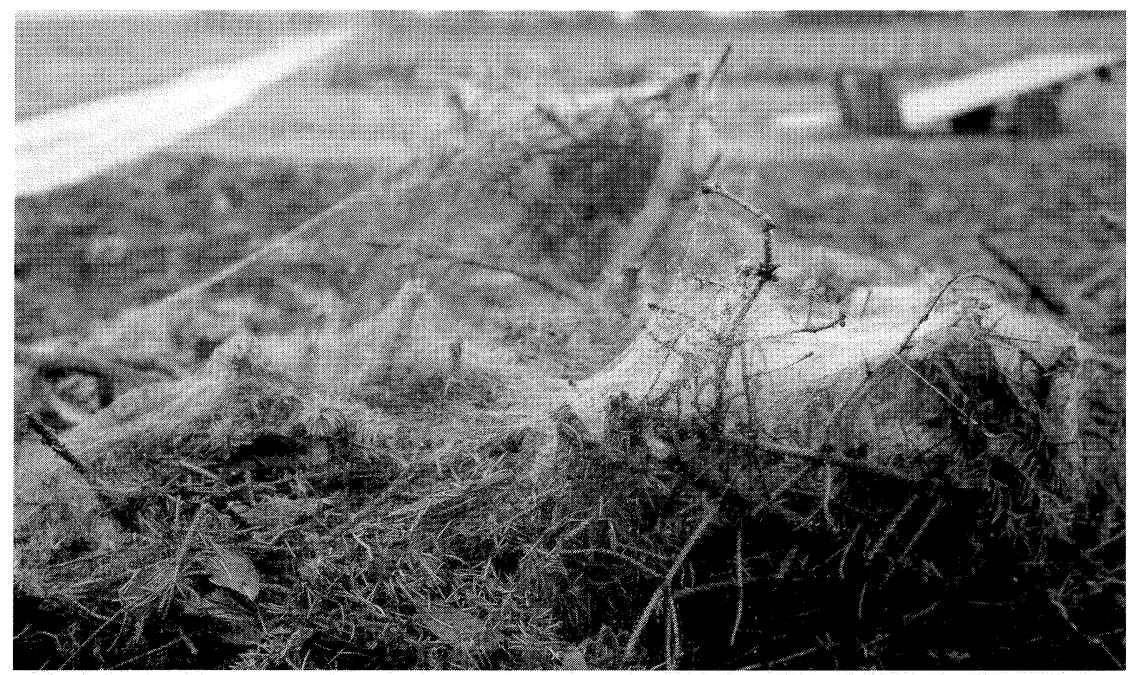

Abbildung 5: Aggregatives Netz auf Zweigen einer gefällten Tanne in Mainz, 3.12.2001. Im Netz wurden 11 verschiedenen Spinnenarten nachgewiesen, dominant war Anelosimus vittatus. (Foto: P. JÄGER)

Figure 5: Aggregative web on branches of a cut fir tree in Mainz, 3.12.2001. In the web 11 different spider species were recorded, with the dominant species Anelosimus vittatus. (photo: P. JÄGER)

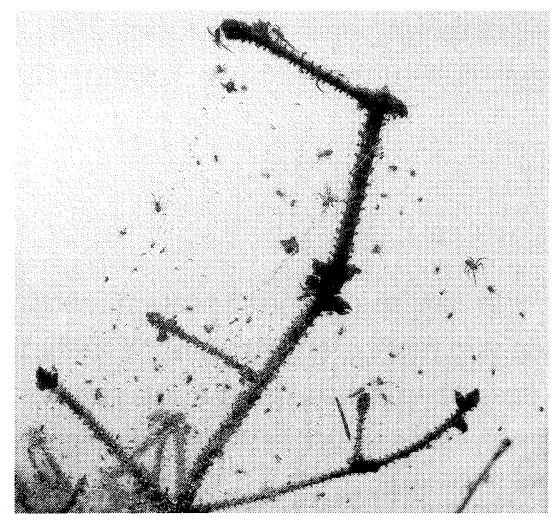

Abbildung 6: Aggregatives Netz auf Zweigen einer gefällten Tanne in Mainz, 3.12.2001. Auf dem Netz sind verschiedene Spinnenarten zu erkennen (Philodromus sp., Clubiona sp.), die als jagende Spinnen bekannt sind. (Foto: P. JÄGER)

Figure 6: Aggregative web on branches of a cut fir tree in Mainz, 3.12.2001. In the web different spider species are recognizable (Philodromus sp., Clubiona sp.), which are known as hunting spiders. (photo: P. JÄGER) 
seidenen Fäden vom Wind verdriften lassen (DECAE 1987). Besonders nach einer kühlen Nacht oder nach einer längeren Periode schlechteren Wetters scheinen die Bedingungen an einem sonnigen Tag für eine solche Verbreitung besonders geeignet $\mathrm{zu}$ sein. Unter optimalen Bedingungen sind Massenauftreten meist einiger weniger Arten keine Seltenheit(DUFFEY \& GREEN 1975, DUFFEY 1998). Ein solches Massenauftreten könnte zu den aus Markgröningen beobachteten Netzen geführt haben: Hunderte von Individuen strömen auf erhabene Punkte (auf dem sonst niedrig bewachsenen Trockenrasen waren dies die höher aufragenden Umbelliferen), um so einen guten Ausgangspunkt für ihren Fadenflug zu haben. Jede Spinne produziert dabei ihren Sicherheitsfaden und allmählich entstehen ganze Netze, deren Dichte nach den jeweils höchsten Punkten zunimmt. Die Beobachtung wurde offenbar zu einem Zeitpunkt gemacht, zu dem der Großteil der Tiere bereits gestartet war.

Ein ähnliches Verhalten wurde bei juvenilen Atypus-Individuen in China (Sichuan Provinz: Emei-Shan) beobachtet: in einer Teeplantage, in der sich im Boden dicht an den Teesträuchern Schläuche einer Atypus-Art befanden, konnten Dutzende von Jungspinnen auf einem Teestrauch bei der aeronautischen Verbreitung beobachtet werden. Dabei hinterließen sie einen feinen Teppich von Seidenfäden auf der Vegetation (JÄGER, unpubl. Beobachtung). Lokale Häufungen von Eigelegen könnten damit einen gewissen Einfluss auf das hier beschriebene Phänomen aggregativer Netze haben.

Das Spinnennetz, welches in Mainz von verschiedenen Spinnenarten auf abgeschnittene Fichtenzweigen appliziert wurde, entspringt wahrscheinlich einer Notreaktion der Spinnen, die den Baum vorher bewohnten. Zunächst kann man davon ausgehen, dass die auf den Zweigen gefangenen Spinnen nicht vom umliegenden Acker stammten. Dafür spricht das Artenspektrum, daß sich vor allem aus typischen Arten der Baum- und Strauchschicht zusammensetzt.

Eine Untersuchung einer in der Nähe stehenden Kiefer zeigte eine ähnliche Zusammensetzung wie die der vorliegenden Untersuchung. Barberfallenfänge von umliegenden Feldern zeigten so gut wie keine Übereinstimmung in der Artzusammensetzung.

Wahrscheinlich versuchten die in den Zweigen siedelnden Spinnen durch die Störung (Fällung plus einsetzender Regen) aus ihren Ruhepositionen getrieben - wieder in höhere Straten des Baumes zu gelangen. 
Dafür spricht die Tatsache, dass gerade auf den erhöhten Punkten das Netz dichter war als an niedrigeren Bereichen. Bei der Artzusammensetzung fällt die hohe Abundanz von Anelosimus vittatus auf. Für diese Art ist eine Präferenz von Strauch- und Baumschicht bekannt (WIEHLE 1937).

In den folgenden Tagen wurden immer noch Individuen beobachtet, die sich an den Spitzen des Haufens einfanden. Es ist nicht auszuschließen, dass über Tausend Individuen von $A$. vittatus die einzeln stehende Tanne besiedelten. $\mathrm{Ob}$ solche Besiedlungsdichten normal sind oder ob sie der unnatürlichen Einzellage des Baumes oder eines starken Lausbefalls (der Baum zeigte große Bereiche mit braunen Nadeln) entspringen, bleibt offen.

Der von HEER (1997) geschilderte Fall ähnelt dem vorstehenden insofern, als dass das von ihm beschriebene aggregative Netz auch auf abgeschnittenen Zweige von Nadelbäumen gewebt wurde. Das könnte ein Hinweis darauf sein, dass Troxochrus nasutus Nadelbäume in hohen, bisher nicht erkannten Abundanzen besiedelt.

Das aggregative Netz von Ostearius melanopygius auf zwei Haufen von Gartenabfällen lässt Fragen offen. Woher stammen die vielen Individuen: vom umliegenden Acker oder aus Gärten, aus denen der Abfall stammte? Warum entsteht anscheinend nur zu einem bestimmten Zeitpunkt ein solch großes Gewebe? Ist es auch eine Reaktion auf eine anthropogene Störung wie bei der Tannenfällung in Mainz, wobei hier ehemals im Garten angesiedelten Spinnen versuchen, ein geeignetes Habitat aufzusuchen? Oder sind es gewisse thermische Faktoren, die über dem teils gärenden Haufen geeignete Bedingungen für einen Start am Fadenfloß vortäuschen, so dass Tausende Spinnen aus umgebenden Bereichen zur Neubesiedlung aufbrechen?

Ostearius melanopygius wird in der Literatur als häufiger Aeronaut bezeichnet (ROBERTS 1987). Als besiedelte Habitate werden in der Literatur ausschließlich synanthrope Bereiche oder benachbarte Habitate angegeben (Gebäude, Gärten, Gärtnereien, Komposthaufen, Schuttplätze; ROBERTS 1987, HEIMER \& NENTWIG 1991). Die Art wurde zumindest im Saarland bzw. in Rheinland-Pfalz auch in natürlichen oder naturnahen Habitaten nachgewiesen (STAUDT, pers. Mitt.). Die Art ist nahezu weltweit verbreitet (MILLIDGE 1985, RUZICKA 1995). Ihr Ursprung wird als Gondwanarelikt von MILLIDGE (1985) in Südamerika vermutet, wo 
verwandte (dort endemische) Arten vorkommen (Laminacauda spp.). Die Verschleppung nach Mitteleuropa könnte die hauptsächlich synanthropen Habitate erklären, die nach dem jetzigen Kenntnisstand in Deutschland besiedelt werden. Ob die beobachteten hohen Abundanzen die Regel bilden oder als Ausnahme auf artifizielle Umstände zurückzuführen sind, muss genauso unbeantwortet bleiben wie die Frage, ob an den aggregativen Netzen im o.g. Fall weitere Arten beteiligt waren.

\section{ZUSAMMENFASSUNG}

In Deutschland kommen große aggregative Netze verschiedener Spinnenarten vor. Die Netze entstehen zufällig und bestehen wahrscheinlich aus den Sicherheitsfäden der einzelnen Individuen. Zwei Hauptursachen könnten für das Entstehen solcher Gewebe verantwortlich sein: 1. Das BallooningVerhalten von Spinnen, die bei optimalen Bedingungen in Massen zu einer aeronautischen Verbreitung aufbrechen und so mit ihren einzelnen Sicherungsfäden Vegetationsstrukturen mit einem Gewebe überziehen können, und 2. Spinnen, die sich bei einer Störung in ihrem Habitat (z.B. das Fällen von Bäumen) aufmachen, um in höhere, früher bewohnte Straten zurückzugelangen.

Eine aktive Aggregation oder ein parasoziales Verhalten kann grundsätzlich nicht ausgeschlossen werden, scheint aber durch die vorliegenden Ergebnisse und Beobachtungen eher unwahrscheinlich. Detaillierte faunistisch-ökologische Untersuchungen von Baumwipfeln könnten zur Klärung dieses Phänomens beitragen. Eine Sammlung von Daten ähnlicher Fälle ist wünschenswert.

Dank: Den Herren Walter MORGENSTERN (Asperg) und Roland KUHN (Mainz) danke ich für die Übermittlung der Funddaten sowie die Erlaubnis, ihre Fotos zu veröffentlichen. Gustavo HORMIGA (Washington) gab Hinweise zur systematischen Einordnung von Ostearius. Aloysius STAUDT (Schmelz) übermittelte dankenswerterweise Daten zu Funden von Ostearius melanopygius bzw. zu dort bewohnten Habitaten. Helmut STUMPF (Würzburg) machte dankenswerterweise Anmerkungen zum Manuskript. 


\section{LITERATUR}

DECAE, A. E. (1987): Dispersal: ballooning and other mechanisms. - In: W. NENTWIG (ed.): Ecophysiology of spiders. - Springer Verlag, Berlin, Heidelberg, New York, London, Paris, Tokyo, 348-356

DUFFEY, E. (1998): Aerial dispersal in spiders. - Proceedings of the 17th European Colloquium of Arachnology, Edinburgh, 1997. (P. A. Selden, ed.), Burnham Beeches, Bucks: Bull. British Arach. Soc., 187-191

DUFFEY, E. \&. M. B. GREEN (1975): A linyphiid spider biting workers on a sewagetreatment plant. - Bull. Br. arachnol. Soc. 3 (5): 130-131

HEER, X. (1997): Beobachtungen zu Troxochrus nasutus (Araneae: Linyphiidae). - Arachnol. Mitt. 14: 81-83

HEIMER, S. \& W. NENTWIG (1991): Spinnen Mitteleuropas. Ein Bestimmungsbuch. - Verlag Paul Parey, Berlin, Hamburg, 543 S.

MILLIDGE, A.F. (1985): Some linyphiid spiders from South America (Araneae, Linyphiidae). - American Mus. Novitates 2836: 1-87

ROBERTS, M. J. (1987): The Spiders of Great Britain and Ireland. Volume 2. Linyphiidae and Check List., Harley Boks, Colchester, $204 \mathrm{~S}$.

RUZICKA, V. (1995): The spreading of Ostearius melanopygius (Araneae: Linyphiidae) through Central Europe. - Eur. J. Entomol. 92 (4): 723-726

WIEHLE, H. (1937): 26. Familie. Theridiidae oder Haubennetzspinnen. In: F. DAHL: Die Tierwelt Deutschlands und der angrenzenden Meeresteile. 33. Teil, Spinnentiere oder Arachnoidea. VIII: Gnaphosidae-Anyphaenidae-Clubionidae-Hahnidae-ArgyronetidaeTheridiidae. Verlag Gustav Fischer, Jena, 119-222

Dr. Peter JÄGER, Institut für Zoologie, Johannes Gutenberg-Universität, D-55099 Mainz, e-Mail: jaegp000@mail.uni-mainz.de 